\title{
Polydiacetylene-based sensors for the activity assay of phospholipase D
}

\author{
Zhijie Zhang, Xiaoqiang Chen \\ State Key laboratory of Materials-Oriented Chemical Engineering, College of Chemical Engineering, , \\ Nanjing Tech University, Nanjing 210009, China \\ chenxq@njtech.edu.cn
}

\begin{abstract}
:
Phospholipase D (PLD) is an important enzyme in regulating biological processes. A polydiacetylene (PDA) with imidazolium as head group was developed as the sensor of PLD activity in this work. PLD catalyzed the hydrolysis reaction of L-a-phosphatidylcholine to produce phosphatidic acid, the negative charges of phosphatidic acid interacted with positive charges of imidazolium, which caused the color transition from blue to red and turn-on fluorescence. No obvious changes of PDAs were observed in absence of PLD or in the presence of PLD coexisting inhibitor. Furthermore, paper based PDAs sensor was constructed and exhibited convenient and sensitive detection for PLD activity.
\end{abstract}

Key words: polydiacetylene, fluorescent and colorimetric sensor, activity assay, phospholipase D

\section{Introduction}

Phospholipase D (PLD) is an important cellular enzyme that widely found in animals, plants, and microorganisms. In consideration of the various functions of PLD in regulating biological processes, developing feasible fluorescence probes for sensing PLD are highly necessary. Polydiacetylenes (PDAs) with conjugated eneyne backbone have received popularly attention due to unique optical properties. Upon the stimulus of the external environment, PDAs undergo blue-to-red color transition accompanied with the appearance of fluorescence. ${ }^{[1]}$ In this study, PDAs with imidazolium as head group was developed for the detection of PLD activity. Upon the PLDcatalyzed hydrolysis reaction of $L-\alpha-$ phosphatidylcholine occurred, PDAs underwent color transition from blue to red and fluorescence enhancement on account of the interaction between PDAs with positive charges and hydrolysis products with negative charges. In the presence of inhibitor, the PLD activity was inhibited, the color and fluorescence changes were prevented simultaneously. All of the changes of PDAs above mentioned were monitored in the process of detecting PLD activity.

\section{Materials and Methods}

The monomer was prepared according to the previous report. $^{[2]}$ To make conjugated PDA vesicles, monomer was dissolved in HEPES buffer $(10 \mathrm{mM}, \mathrm{pH} 7.4)$ to afford a total monomer concentration of $1 \mathrm{mM}$. The solution was then sonicated at $80{ }^{\circ} \mathrm{C}$ for $30 \mathrm{~min}$. The vesicle solution was filtered through a $0.8 \mu \mathrm{m}$ filter and the filtrate was cooled at $4{ }^{\circ} \mathrm{C}$ for at least $12 \mathrm{~h}$. Polymerization was carried out at 4 ${ }^{\circ} \mathrm{C}$ by irradiating the solution $254 \mathrm{~nm}$ UV light (1 $\mathrm{mW} / \mathrm{cm}^{2}$ ) for $3 \mathrm{~min}$.

The activity assay of PLD was performed as follows. Stock solutions of $1 \mathrm{mM}$ of PDAs (according to the monomer concentration) in HEPES buffer (10 mM, pH 7.4), $4 \mathrm{U} / \mathrm{mL}$ PLD in Tris- $\mathrm{HCl}$ buffer (10 mM, pH 8.0), $1 \mathrm{mM}$ of L-aPhosphatidylcholine in methanol and $1 \mathrm{mM}$ of halopemide (an inhibitor of PLD) in DMSO were prepared separately. In a typical experiment, test solution was prepared by placing $30 \mu \mathrm{L}$ of the PDA solution into a test tube and diluted to $3 \mathrm{~mL}$ with HEPES buffer (10 mM, pH 7.4). Then various equivalents of analytes were added to the test solution, all the fluorescence and UVvis absorption measurements were collected after $15 \mathrm{~min}$. Excitation and emission were at $492 \mathrm{~nm}$ and $563 \mathrm{~nm}$, respectively.

To prepare paper test strips loaded with PDAs, $8 \mu \mathrm{L}$ of stock solution of PDA vesicles (1 $\mathrm{mM}$ ) was dropped on filter paper diameter of 6 $\mathrm{mm}$, until the paper was dried at room temperature. Then the paper was immersed in 
the solution of L-a-phosphatidylcholine with different concentrations PLD.

\section{Results and discussion}

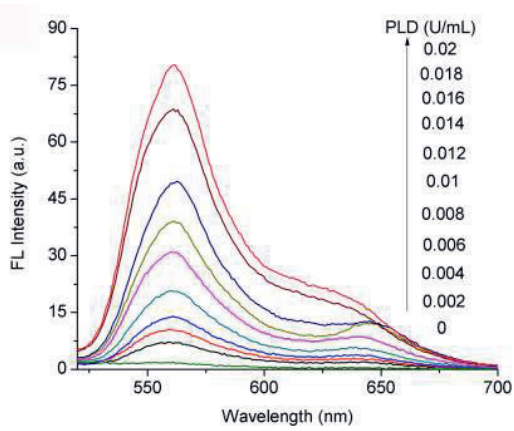

Fig. 1 Fluorescence intensity spectra changes of PDAs $(10 \mu M)$ containing L- $\alpha$-phosphatidylcholine $(10 \mu M)$ in the presence of different amounts of PLD ranging from $0 \mathrm{U} / \mathrm{mL}$ to $0.02 \mathrm{U} / \mathrm{mL}$.

The fluorescence intensity changes with different amounts of PLD were studied. L- $\alpha$ phosphatidylcholine was firstly added to the polymer solution, then the fluorescence emission intensity at $563 \mathrm{~nm}$ increased with the increasing amounts of PLD which could result in the hydrolysis of L-a-phosphatidylcholine, and the fluorescence intensity enhanced about 46-fold with $0.02 \mathrm{U} / \mathrm{mL}$ of PLD after $15 \mathrm{~min}$ (Figure 1). The fluorescence intensity was linear with the amount of PLD ranging from 0 $\mathrm{U} / \mathrm{mL}$ to $0.008 \mathrm{U} / \mathrm{mL}$, therefore, the detection limit (DL) of the PDAs containing L- $a-$ phosphatidylcholine toward PLD was calculated as $0.0006 \mathrm{U} / \mathrm{mL}$, which means that this sensor has the great sensitivity toward the activity of PLD.

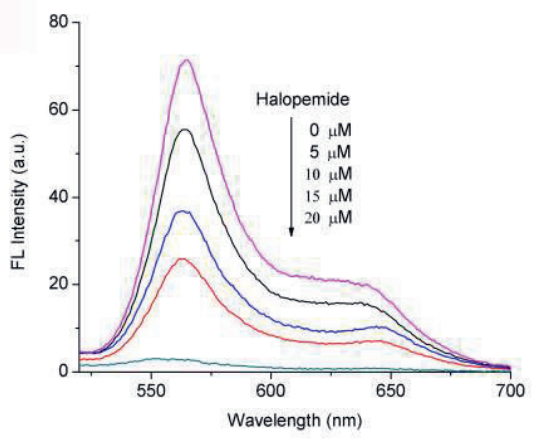

Fig. 2. Fluorescence emission spectra of PDAs (10 $\mu M)$ containing L- $\alpha$-phosphatidylcholine $(10 \mu M)$ in the presence of PLD $(0.04 \mathrm{U} / \mathrm{mL})$ and different concentrations of halopemide $(0 \mu \mathrm{M}, 5 \mu \mathrm{M}, 10 \mu \mathrm{M}$, $15 \mu M, 20 \mu M)$ in HEPES buffer (10 mM, pH 7.4).

Halopemide, as an inhibitor of PLD, could decrease the activity of PLD and retard the hydrolysis process of L-a-phosphatidylcholine. We pretreated PLD with various concentrations of halopemide for $15 \mathrm{~min}$ at room temperature. Then the mixture was added to PDAs solution containing L-a-phosphatidylcholine. Obviously, from the Figure 2, fluorescence intensity gradually decreased with increasing concentration of halopemide, indicating the definite relationship between fluorescence intensity and PLD activity. On the basis of relationship between inhibition efficiency and concentration of halopemide, the $\mathrm{IC}_{50}$ value of halopemide toward PLD activity was estimated to be $10.8 \mu \mathrm{M}$.

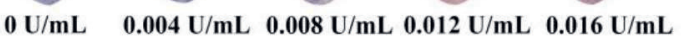

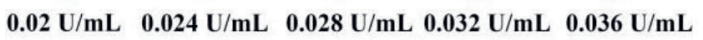

Fig. 3. Pictures of PDAs-loaded test strips, treated with L- $\alpha$-phosphatidylcholine and different concentrations of PLD.

In order to investigate the practical application of PDAs for detecting PLD activity, we futher made paper-based test strips via loading PDAs to filter paper strips. After these test strips were exposed to the solution of $L-\alpha-$ phosphatidylcholine and different concentrations of PLD, the obvious color transitions could be observed by naked eye. With increasing concentration of PLD, the color of PDAs-based paper transferred from blue to red gradually, indicating that PLD could be detected successfully (Figure 3 ). Therefore, the PDAs-loaded test strips could be used as a sensitive and efficient tool with simple making process for the detection of PLD.

\section{Conclusion}

We developed a fluorescence probe based on PDAs for the detection of PLD. PLD catalyzes the hydrolysis of L-a-phosphatidylcholine and leads to the blue to red color change and turnon fluorescence response. The color and fluorescence changes can be inhibited by the inhibitor (halopemide) of PLD. The probe can be further to realize the convenient and sensitive detection of PLD by naked eye via loading the PDAs to filter paper strips.

\section{References}

[1] X. Chen, G. Zhou, X. Peng, Juyoung Yoon, Biosensors and chemosensors based on the optical responses of polydiacetylenes, Chem. Soc. Rev. 41, 4610-4630 (2012); doi: $10.1039 / \mathrm{c} 2 \mathrm{cs} 35055 \mathrm{f}$

[2] X. Chen, S. Kang, M. J. Kim, J. Kim, Y. S. Kim, H Kim, B. Chi, S.-J. Kim, J. Y. Lee, J. Yoon, ThinFilm Formation of Imidazolium-Based Conjugated Polydiacetylenes and Their Application for Sensing Anionic Surfactants, Angew. Chem. Int. Ed. 49, $1422 \quad-1425 \quad$ (2010); doi: 10.1002/anie.200905041 